\title{
Umweltstudien mit Smartphone für Schüler*innen am Beispiel der Untersuchung der Luft in Bienenstöcken
}

\author{
Sebastian Höfner, Andreas Schütze \\ Lehrstuhl für Messtechnik, Universität des Saarlandes, Saarbrücken, Deutschland \\ Kontakt: s.hoefner@Imt.uni-saarland.de
}

\section{Einleitung}

Der Erfolg der sozialen Bewegung „Fridays for Future" zeigt, dass sich Jugendliche mehr denn je für die Themen Klima und Umweltschutz interessieren. Im Rahmen des DBU-Projekts "SUSmobil“ (Schüler-Umwelt-Studien mit mobilen Messgeräten) soll deren Umweltbewusstsein durch das Vermitteln von Grundlagen zu modernen Gassensoren [1] sowie das Anfertigen eigener Umweltstudien gefördert werden. In diesem Projekt wird Schüler*innen die Möglichkeit geboten, eigene Fragestellungen für Umweltstudien zu entwickeln, die mit moderner Sensorik und unterstützt von Wissenschaftler*innen umgesetzt werden.

Aufgrund der Omnipräsens und Mobilität von Smartphone und Tablet sind diese prädestiniert, um Schüler*innen bei der Beantwortung wissenschaftlicher Fragestellungen zu unterstützen. Lernen ist nicht mehr auf feste Orte wie Klassenraum oder Schule im Allgemeinen begrenzt. Mobile Technologie fungiert dabei als Viermitter eines solchen Lernprozesses im Sinne des Ansatzes von „Mobile Assisted Seamless Learning" [2]. Die Vernetzung von Informations- und Kommunikationstechniken durch das Internet of Things (IoT) erlaubt es, jederzeit und überall auf Messdaten zuzugreifen. Apps auf Smartphone, Tablet und Co. erlauben es, Messdaten aufzuzeichnen und intuitiv zu analysieren. Diese Simplifizierung kann dabei helfen, Vorbehalte und Ängste davor, Fehler zu machen, abzubauen, was zu einem besseren Lernerfolg führen kann.

Das Konzept wird im Folgenden am Beispiel einer Umweltstudie zweier Schülerinnen der Klassenstufe 12 mit dem Thema „Untersuchung der Luft in Bienenstöcken" vorgestellt.

\section{Citizen Science für Schüler*innen}

Citizen Science bietet als Kooperation von Bürgern und Wissenschaftler*innen großes Potential für die Gewinnung neuer wissenschaftlicher Erkenntnisse und gewinnt zunehmend an Bedeutung [3].

Dabei können Citizen Science-Projekte wissenschaftliche Ziele wie Datenerfassung und -analyse kombinieren mit formellen und informellen Zielen für den naturwissenschaftlichen Unterricht, öffentlichem Engagement sowie Umweltschutz. Die große Herausforderung dabei ist das Zusammenspiel aus Gestaltung, Verwaltung und Betrieb von Citizen
Science Projekten einerseits und die Sicherstellung wissenschaftlich akzeptabler Daten sowie deren Auswertung andererseits, was ein viel diskutiertes Problem darstellt $[4,5,6]$. Aus diesem Grund ist es wichtig, dass Schüler*innen nicht nur als bloße Datensammler agieren, sondern auch ein fundiertes Grundwissen für das jeweilige Thema aufbauen. Erst dann kann kritisches Hinterfragen der Messdaten erfolgen und dazu führen, dass sich Werte, Einstellungen und Identitäten ändern oder Verhaltensänderungen herbeigeführt werden [7].

Die Klimabewegung "Fridays for Future" hat bei vielen Menschen und insbesondere Schüler*innen das Interesse geschaffen, sich wieder verstärkt mit ihrer Umwelt und ihrem Handeln auseinander zu setzen. Dieses Interesse kann durch speziell für Schüler*innen entworfene Citizen Science Projekte unterstützt werden [8].

Ein Beispiel für ein solches Citizen Science Projekt für Schüler*innen stellt SUSmobil dar, welches das Thema Luftqualität in den Vordergrund rückt. Das Projekt ist in insgesamt vier Module unterteilt, wobei sich zwei zunächst mit den theoretischen Grundlagen der Halbleiter-Gassensorik und zwei mit der Messung von Umweltparametern sowie der Innenund Außenluftqualität auseinander setzen, letzteres in Form von Schülerumweltstudien. Durch diese Aufteilung in theoretische Grundlagenvermittlung und der Möglichkeit zur Erstellung und Beantwortung eigener Umweltfragestellungen wird einerseits das nötige Hintergrundwissen als auch die Motivation zur weitergehenden Beschäftigung mit dem Thema vermittelt. Die ersten drei Module sind als sogenannte "Selbstlernkurse" aufgebaut und richten sich an Schulklassen im Alter zwischen 12 und 18 Jahren. Diese Module behandeln die Funktionsweise und Kalibrierung von Hableiter-Gassensoren sowie die Durchführung von Messungen der Innenraumluftqualität. Darauf aufbauend können Schüler*innen im vierten Modul eigene Umweltfragestellungen entwikkeln und Umweltstudien unterstützt durch Wissenschaftler*innen nach dem Citizen Science Konzept durchführen.

\section{Formales und Informelles Lernen mit Smartphone und Tablet}

Bezugnehmend auf eine Studie von Dahlstrom et al. gewinnen moderne Technologien wie Smartphone und Tablet sowohl im Altag als auch im 
Unterricht immer mehr an Bedeutung [9]. Viele Schulen und Universitäten setzen mittlerweile auf online-Lernresourcen oder nutzen Möglichkeiten der "classroom response", durch die Lernende den Lehrenden über technische Hilfsmittel live Feedback, $z$. B. in Form anonymisierter Umfragen, geben können [10]. Das Angebot reicht dabei von online zur Verfügung gestellten Vorlesungsmaterialien über Lern-Apps bis hin zu virtuellen Laboren [11]. Der Vorteil dieser Lern- und Lehrmedien ist, dass diese dem Lernenden aus dem Alltag gut bekannt und somit authentisch sind [12]. Smartphone und Tablet können somit als Teil des naturwissenschaftlichen Unterrichts eingesetzt werden [13].

Die Möglichkeit, sich auch abseits des Unterrichts ohne formale Vorgaben mit einem wissenschaftlichen Thema auseinanderzusetzen, sorgt dafür, dass Schüler*innen Hemmungen und Ängste verlieren. Smartphone und Tablet können als mobile Labore verwendet werden und so naturwissenschaftliches Lernen im Alltag ermöglichen. Über "Internet of Things" (IOT) Dienste ist es $z$. B. jederzeit möglich, auf Sensordaten zuzugreifen, ohne großen Aufwand eigene Graphen und Wertetabellen anzulegen und so auch zeitlich unabhängiger zu sein. Dadurch wird das Spektrum an Anorderungen innerhalb eines Experiments reduziert und der Fokus kann verstärkt auf das Experiment an sich gelenkt werden [14].

Ein Beispiel für einen kostenlosen loT-Dienst stellt "ThingSpeak" von MathWorks dar. Er bietet die Möglichkeit, Sensordaten mithilfe eines internetfähigen Mikrocontrollers an einern "ThingSpeak"Server zu senden, diese dort zu speichern und anschließend sowohl auf dem Smartphone oder Tablet als auch am PC darzustellen und auszuwerten.
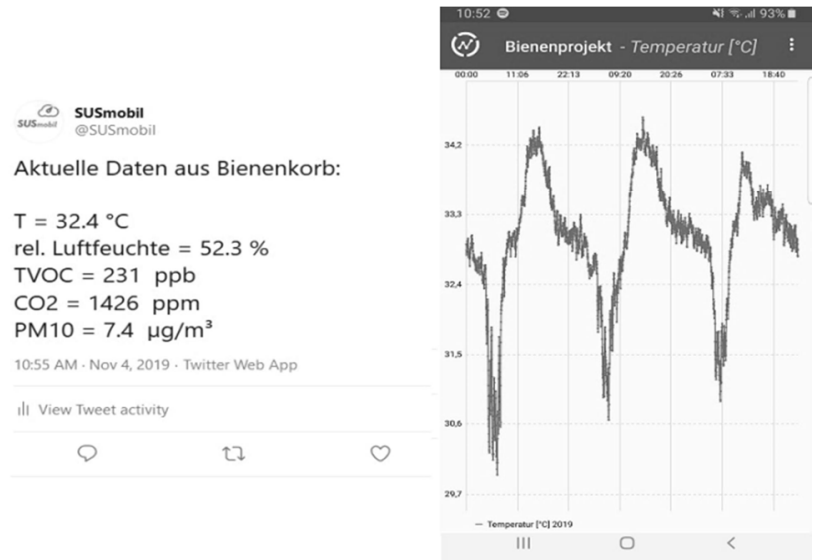

Abb. 1: Links: Automatisch versandter Tweet; Rechts: Darstellung von Sensordaten über die Smartphone App "ThingSpeak“"

Abbildung 1 zeigt zwei Beispiele zur Darstellung von Sensordaten über eine Smartphone App und einen automatisiert generierten Tweet, der über aktuelle Messdaten informiert.
Gerade der Einbezug von Social Media eröffnet den Citizen Scientists neue Wege, ihre Forschung zu präsentieren und andere für wissenschaftliche Themen zu begeistern. Wissenschaft erhält dadurch auch einen sozialen Aspekt, der dafür sorgt, dass Forschung verständlich und zielgruppengerecht vermittelt wird. Gleichzeitig wird die Motivation gestärkt.

\section{Schülerumweltstudie - Untersuchung der Luftzusammensetzung in Bienenstöcken}

In Zusammenarbeit mit dem Landesverband saarländischer Imker und zwei Schülerinnen der Klassenstufe 12 des Geschwister-SchollGymnasiums in Lebach, wurde ein „Jugend forscht"Projekt zur Untersuchung von Bienenstöcken auf Gase, Feinstaub, Temperatur und Luftfeuchte konzipiert. Es wird dadurch die Möglichkeit geboten, an einem authentischen und hochaktuellen Kontext wissenschaftlich $\mathrm{zu}$ arbeiten, indem Daten aufgenommen, ausgewertet und interpretiert werden. Die erforderlichen theoretischen Grundlagen zur Funktionsweise von Gassensoren, deren Kalibrierung und Datenauswertung sowie -interpretation können sich die Schüler*innen in den oben skizzierten Selbstlernkursen, entwickelt von Wissenschaftler*innen und Didaktiker*innen, erarbeiten. Auch während der Durchführung der Umweltstudie stehen jederzeit Wissenschaftler*innen unterstützend zur Seite.

\section{Fragestellungen}

Um zu entscheiden, welchen konkreten Fragestellungen die Schülerinnen nachgehen wollen, führten sie zunächst eine Literaturrecherche durch. Grundlage hierzu stellten die Arbeiten von Seeley [15], Starks et al. [16], Sachs et al. [17], Negri et al. [18] und Doull [19] dar. Die ausgewählten Fragestellungen setzen sich dabei aus der Bestätigung vorangegangener Untersuchungen sowie neuer Fragestellungen zusammen:

- Welche Temperatur und Luftfeuchte herrscht typischerweise in einem Bienenstock?

- Sind diese unabhängig von äußeren Bedingungen?

- Steigt die Feinstaubbelastung, wenn Bienen Blütenstaub sammeln und in den Bienenstock bringen?

- Wie hoch sind die Gesamt-VOC- und $\mathrm{CO}_{2-}$ Konzentrationen in einem bewohnten im Vergleich zu einem leeren Bienenstock?

- Lüften Bienen oberhalb eines Schwellwertes der $\mathrm{CO}_{2}$-Konzentration aktiv?

\section{Experimenteller Aufbau und verwendete Sensoren}

Der experimentelle Aufbau besteht aus einem bewohnten und einem leeren Bienenkorb, siehe 
Abb. 2. Der leere Bienenkorb dient zur Aufnahme von Referenzdaten, um den Einfluss von ausdünstendem Holz zu quantifizieren.

Bei den verwendeten Sensoren handelt es sich um einen Halbleiter-Gassensor SGP30, der die Summe aller flüchtigen organischen Verbindungen (TVOC) misst, einen nicht-dispersiven Infrarotabsorptionssensor SCD30 mit integriertem Temperatur- und Luftfeuchtigkeitsmodul zur Messung von $\mathrm{CO}_{2}$, Temperatur und Luftfeuchte und einen Feinstaubsensor SPS30, basierend auf Laserstreuung, zur Messung der Feinstaubfraktionen PM2.5 und PM10. Alle Sensoren stammen von der Firma Sensirion.

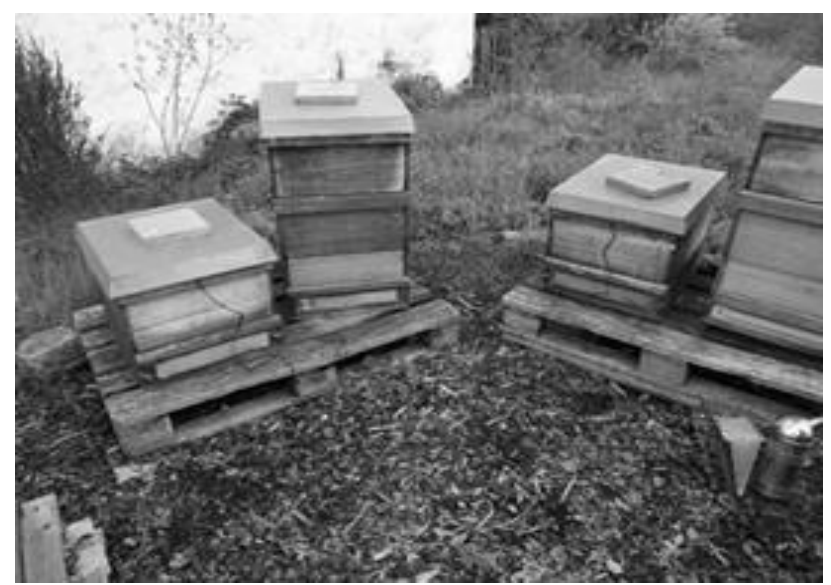

Abb. 2: Verwendete Bienenstöcke für die Umweltstudie. Links: Unbewohnter Stock; Rechts: Bewohnter Stock

Gesteuert und ausgelesen werden die Sensoren über den internetfähigen Mikrocontroller ESP8266, der die Messdaten über eine WLAN-Verbindung an den ThingSpeak-Server sendet. Der komplette Messaufbau wurde in einem Bienenrahmen befestigt und in der Mitte des Bienenkorbs platziert. Um die Sensoren vor direktem Kontakt mit den Bienen zu schützen, wurde ein Drahtgeflecht um sie gespannt. Da Bienen dazu neigen, größere Öffnungen mit Wachs zu verschließen, wurde darauf geachtet, dass das Drahtgeflecht sehr feinmaschig ist.

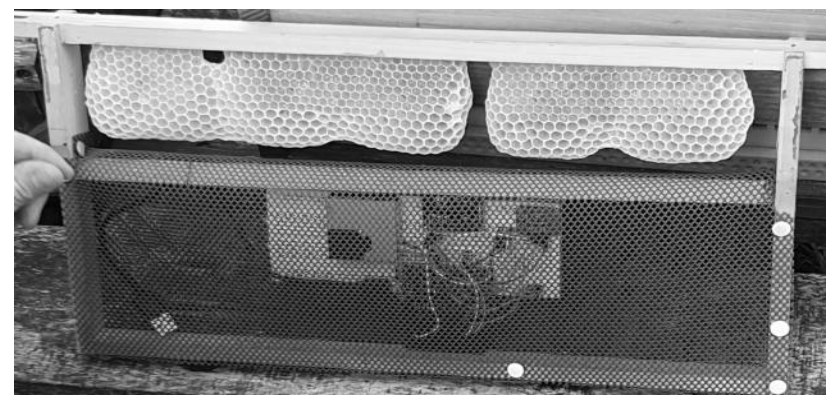

Abb. 3: Versuchsaufbau mit den verwendeten Sensoren

Der zeitliche Abstand zwischen zwei Messungen beträgt ca. 90 Sekunden. Seit dem 14.04.2019 nimmt das System, bis auf eine dreitägige Ausnahme aufgrund einer Störung der Internetverbindung, seitdem ununterbrochen Messdaten auf.
Diese sind öffentlich zugänglich auf der Webseite www.susmobil.de.

\section{Datenaufnahme und Auswertung}

Zur Auswertung soll hier beispielhaft der Zeitraum vom 15.07. bis einschließlich 23.07.2019 betrachtet werden, d.h. über eine Woche. Die Verläufe der Messdaten sind prinzipiell bis auf kleinere Abweichungen in allen Zeitintervallen ähnlich.

\section{Temperatur}

In Abbildung 4 sind die Temperaturverläufe dargestellt. Die Temperatur im leeren Vergleichskorb folgt wie zu erwarten einem deutlichen Tag-Nacht-Rhythmus. Auch im bewohnten Korb ist ein Rhythmus zu erkennen, wenn auch deutlich weniger ausgeprägt. Vor allem ist er gegenläufig zum Verlauf der Außentemperatur, da die Temperatur in der Nacht leicht ansteigt und tagsüber abfällt. Diese Beobachtung kann dadurch erklärt werden, dass in der Nacht mehr Bienen im Stock sind, diesen daher zusätzlich aufwärmen. Am Tag wird die Temperatur von den Bienen durch Fächern ihrer Flügel regulieren.

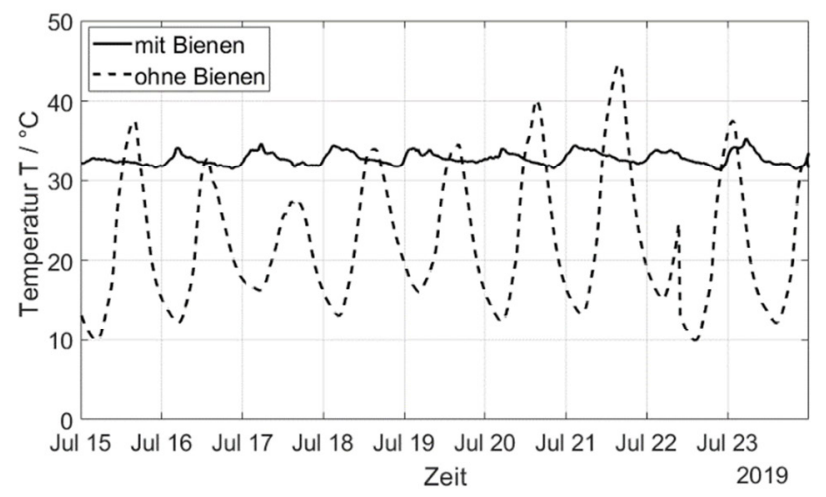

Abb. 4: Verlauf der Temperatur in Bienenkörben mit (durchgezogene) und ohne Bienen (gepunktete Linie)

Die durchschnittlich gemessene Temperatur betrug $33,0^{\circ} \mathrm{C}$ mit einer Standardabweichung von $0,8^{\circ} \mathrm{C}$, wobei minimal $31,0^{\circ} \mathrm{C}$ und maximal $35,5^{\circ} \mathrm{C}$ gemessen wurden. Damit liegt die Temperatur um ca. $2{ }^{\circ} \mathrm{C}$ niedriger als in der Messung von Starks et al. [20] innerhalb des Brutkerns, was durch die Platzierung der Sensoren knapp unter und nicht exakt im Kern erklärt werden kann. Die Temperatur hängt nur sehr geringfügig von den äußeren Bedingungen ab.

\section{Relative Luftfeuchte}

Abbildung 5 zeigt einen Vergleich der relativen Luftfeuchte mit und ohne Bienen, wobei zu berücksichtigen ist, dass diese auch von der Temperatur abhängt. Die mittlere relative Luftfeuchte im Korb mit Bienen beträgt $50,5 \%$ mit einer Standardabweichung von $4,1 \%$, wobei minimal $38,6 \%$ und maximal 60,2 \% gemessen wurden. Im Vergleich weist die relative Feuchte im Referenzkorb höhere 
Schwankungen auf (im Mittel $49 \%$, Standardabweichung $10,4 \%$, min. $30,4 \%$, max. $71,7 \%$ ).

Die Ergebnisse stimmen mit den Beobachtungen von Starks et al. [16] überein, der eine durchschnittliche relative Luftfeuchte zwischen 45 und $55 \%$ gemessen hat. Auch hier scheinen die Messwerte nicht von den äußeren Bedingungen abzuhängen, was für eine aktive Regulierung der Feuchte durch die Bienen spricht.

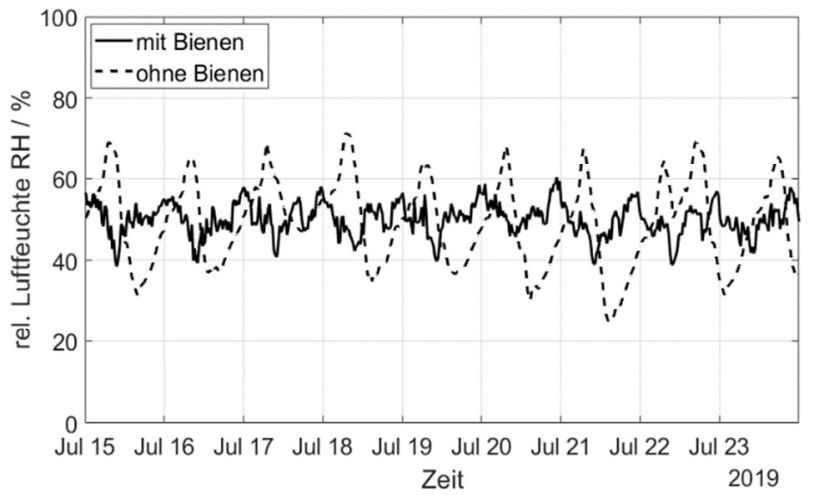

Abb. 5: Verlauf der relativen Feuchte in Bienenkörben mit (durchgezogene) und ohne Bienen (gepunktete Linie)

Die Luftfeuchte hat einen direkten Einfluss auf das Brutverhalten. Nach Doull [19] schlüpfen unterhalb einer relativen Luftfeuchte von $50 \%$ keine Larven mehr, weshalb eine Regulierung zwingend für den Fortbestand des Volkes ist.

\section{Feinstaub PM10 und PM2.5}

Die Untersuchungen der Feinstaubkonzentration innerhalb des Bienenkorbs zeigen keinen eindeutigen periodischen Verlauf. Der Mittelwert liegt mit $6,0 \mu \mathrm{g} / \mathrm{m}^{3}$ (Standardabweichung $2,5 \mu \mathrm{g} / \mathrm{m}^{3}$ ) auf niedrigen Niveau [20], sodass zumindest für diesen Zeitraum im Siommer ausgeschlossen werden kann, dass sich die Luft im Bienenkorb mit (Blüten-)Staub anreichert. Dieser ist entweder an den Bienen selbst oder in den Waben gebunden.

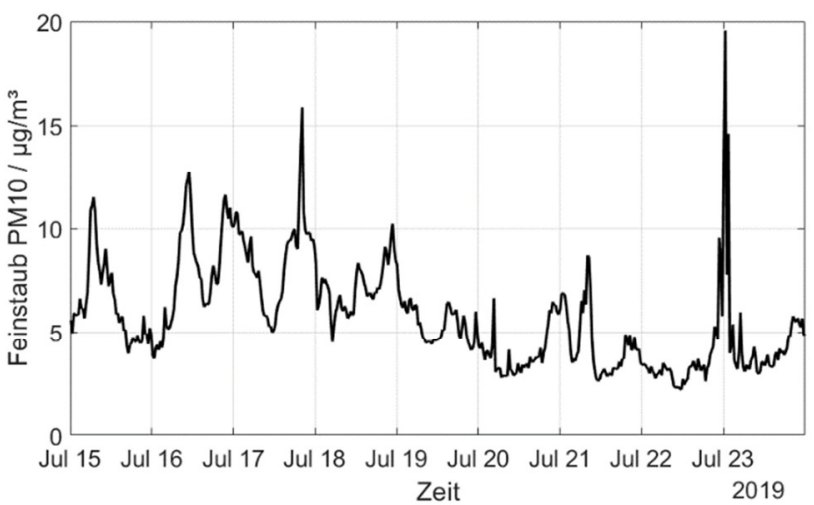

Abb. 6: Verlauf der Feinstaub-Konzentration PM10 $($ Partikel $<10 \mu \mathrm{m})$ in einem Bienenkorb mit Bienen
Diese Vermutung stützt sich auf Untersuchungen von Negri et al. [18], die gezeigt haben, dass sich Feinstaub in den feinen Haaren der Bienen festsetzt.

Aufgrund von technischen Problemen mit dem Feinstaubsensor im Kontrollkorb stehen keine Vergleichsdaten zur Verfügung. Zwischen den PM2.5 und PM10 Messwerten zeigte sich kein signifikanter Unterschied, weshalb auf die Darstellung der PM2.5 Werte verzichtet wird.

\section{TVOC}

Der Vergleich der TVOC-Konzentration mit und ohne Bienen in Abbildung 7 zeigt jeweils einen periodischen Verlauf in einem 24-Stunden-Rhythmus. Die Konzentration innerhalb des Bienenstocks mit Bienen liegt um ca. $800-1000 \mathrm{ppb}$ höher. Zudem sind beide Verläufe, ähnlich wie die Temperatur, s. Abb. 4, um ca. 6 - 12 Stunden zeitversetzt. Während die TVOC-Konzentration im Stock ohne Bienen am Tag ansteigt, fällt sie im Korb mit Bienen tagsüber und steigt nachts wieder an.

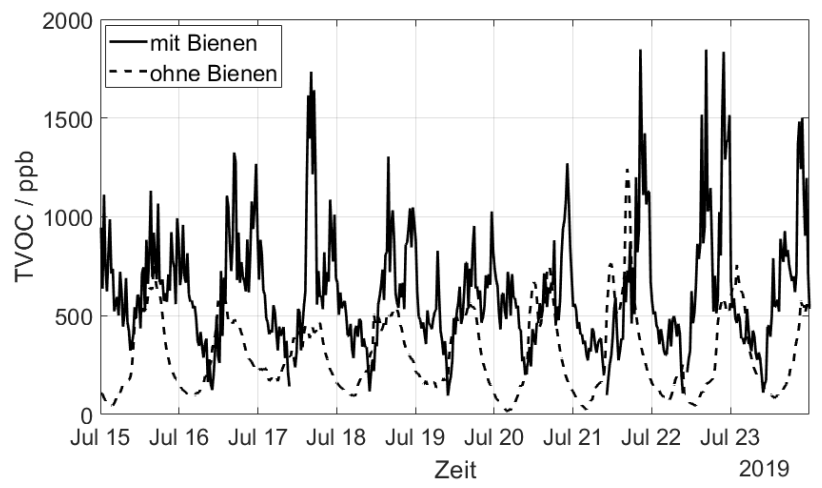

Abb. 7: Verlauf der TVOC-Konzentration in Körben mit (durchgezogene) und ohne Bienen (gepunktete Linie)

Dies kann zum einen durch die größere Zahl der Bienen in der Nacht bzw. auch durch eine verstärkte Ventilation durch Flügelschlagen tagsüber erklärt werden. Auf der anderen Seite sorgt die erhöhte Temperatur tagsüber dafür, dass vermehrt flüchtige organische Verbindungen aus den Holzkästen ausdunsten, was zu einer Erhöhung der TVOCKonzentration in dem Korb ohne Bienen führt.

$\mathrm{CO}_{2}$

Analog zur TVOC-Konzentration zeigt auch der Verlauf der $\mathrm{CO}_{2}$-Konzentration ein periodisches Verhalten, s. Abb. 8. Im vorliegenden Zeitraum erreicht diese einen täglichen Maximalwert zwischen 6.000 und $8.000 \mathrm{ppm}$ zwischen 10 und $12 \mathrm{Uhr}$ vormittags im bevölkerten Korb. In anderen Zeiträumen wurden sogar Maximalwerte von bis zu 14.000 ppm gemessen. Der Referenzwert im leeren Korb liegt dagegen nahe dem natürlichen Hintergrundwert von 400 ppm. Aufgrund der periodischen Verläufe der TVOC- und $\mathrm{CO}_{2}$-Konzentration liegt die Vermutung nahe, dass 
Bienen diese durch vermehrtes Flügelschlagen und die dadurch entstehende Ventilation regulieren.

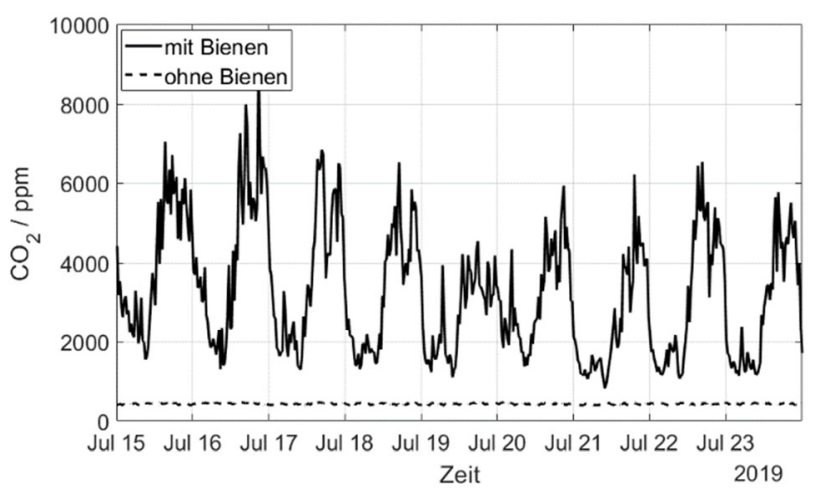

Abb. 8: Verlauf der $\mathrm{CO}_{2}$-Konzentration in Bienenkörben mit (durchgezogene) und ohne Bienen (gepunktete Linie).

Nach Seeley [15] besitzen Bienen $\mathrm{CO}_{2}$-Rezeptoren und sind damit in der Lage, verschiedene $\mathrm{CO}_{2}$-Konzentration $\mathrm{zu}$ unterscheiden. $\mathrm{CO}_{2}$ fungiere dabei einerseits als Indikator für die Abwesenheit von Sauerstoff und sei andererseits verantwortlich für eine signifikante Hemmung des Enzymkomplexes Succinatdehydrogenase des Citratzyklus. Außerdem seien die physiologischen Probleme der optimalen Aufrechterhaltung des $\mathrm{pH}$-Wertes und der Wasserretention in einer Umgebung mit höherer $\mathrm{CO} 2$ Konzentration größer, weshalb eine Regulierung notwendig sei.

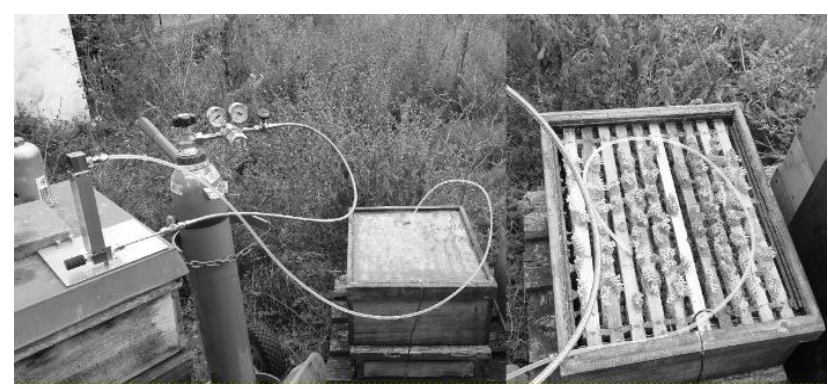

Abb. 9: Experimenteller Aufbau zum Testen, ob Bienen $\mathrm{CO}_{2}$ aktiv regulieren.

Da die gemessenen Konzentrationabfälle jedoch immer am späten Vormittag auftreten, könnte es auch sein, dass die Bienen lediglich auf die sich erwärmende Außenluft reagieren. Um den Einfluss der Temperatur auszuschließen, wurde daher die $\mathrm{CO}_{2}$-Konzentration künstlich innerhalb von wenigen Minuten durch Einleiten von $\mathrm{CO}_{2}$ aus einer Gasflasche erhöht. Der Versuchsaufbau ist in Abbildung 8 gezeigt. Es wurde darauf geachtet, dass die $\mathrm{CO}_{2-}^{-}$ Konzentration $4 \%$ nicht überschreitet, um die Bienen nicht zu gefährden.

In Abbildung 10 ist jeweils der Verlauf der $\mathrm{CO}_{2-}$ Konzentration über einen Zeitraum von 200 Sekunden aufgetragen bei externer Zugabe von $\mathrm{CO}_{2}$ mit den Flussdichten $0,5 \mathrm{l} / \mathrm{min}, 7 \mathrm{l} / \mathrm{min}$ und $12 \mathrm{l} / \mathrm{min}$ gezeigt. Die Ergebnisse in Abb. 10 zeigen deutlich, dass Bienen auf das $\mathrm{CO}_{2}$ reagieren und die Frequenz ihrer Flügelschläge solange anpassen, bis die Lüftungsrate ausreicht, um die Konzentration auf einen Wert zwischen 15.000 und $20.000 \mathrm{ppm}$ herunterzuregeln. Dieser Wert kann als Schwellwert interpretiert werden, oberhalb dessen die Bienen aktive Lüftung betreiben. Erst ab einer Flussdichte von $12 \mathrm{l} / \mathrm{min}$ sind die Bienen nicht mehr in der Lage, die $\mathrm{CO}_{2}$-Konzentration zu regulieren.

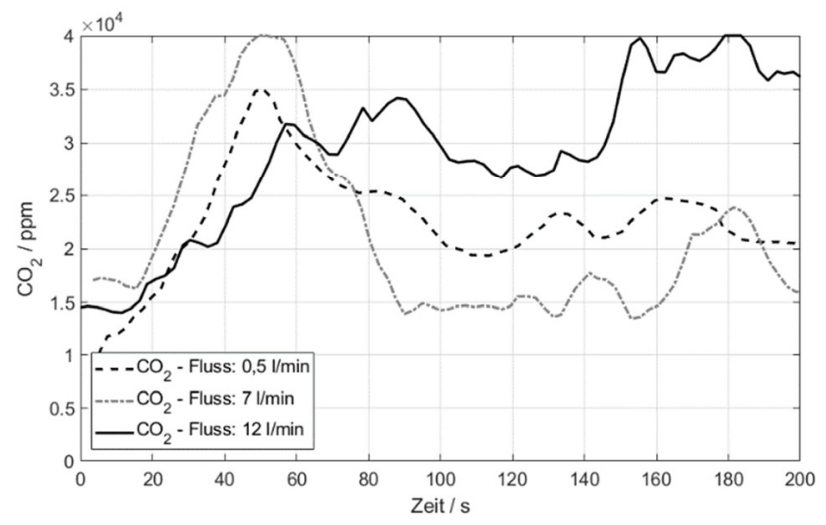

Abb. 10: Verlauf der $\mathrm{CO}_{2}$-Konzentration nach Einlass von $\mathrm{CO}_{2}$ mit Flussraten von $0,5,7$ und $12 \mathrm{l} / \mathrm{min}$.

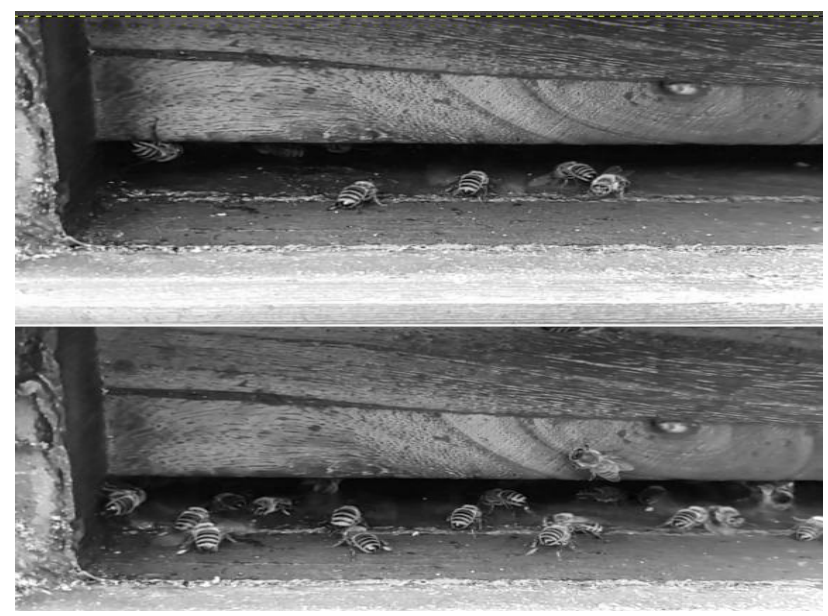

Abb. 11: Reaktion der Bienen auf aktives Einlassen von $\mathrm{CO}_{2}$; Flussraten $5 \mathrm{l} / \mathrm{min}$ (oben) und $12 \mathrm{l} / \mathrm{min}$ (unten)

In Abbildung 11 is zu sehen, dass sich abhängig vom $\mathrm{CO}_{2}$-Fluss mehr Bienen mit den Flügeln nach außen an einem Ausgang des Bienenkorbs sammeln, um eine Ventilation des Korbs zu erreichen.

Dieses Verhalten zeigte sich ab einem Fluss von $5 \mathrm{l} / \mathrm{min}$. Davor war lediglich ein Anstieg der Lautstärke der Summgeräusche zu vernehmen. Aus diesen Beobachtungen ist abzuleiten, dass Bienen das $\mathrm{CO}_{2}$ wahrnehmen können und aktiv regulieren.

\section{Fazit und Ausblick}

Es konnte gezeigt werden, dass Temperatur, TVOC- und $\mathrm{CO}_{2}$-Konzentrationen in einem Bienenstock einem periodischen Verlauf folgen. Die Luft- 
feuchte hingegen blieb nahezu konstant und war unabhängig von den äußeren Bedingungen. Die Feinstaubkonzentration zeigt keine Auffälligkeiten.

Durch aktives Einlassen von $\mathrm{CO}_{2}$ war es möglich nachzuweisen, dass Bienen dieses wahrnehmen und regulieren können. Dies scheint oberhalb eines Schwellwertes von 15.000 bis $20.000 \mathrm{ppm}$ zu erfolgen. Interessant ist in diesem Zusammenhang, dass der Mensch Luft bereits ab einer Konzentration von 1.000 ppm (Pettenkofer-Zahl) als "schlecht" bzw. „verbraucht" empfindet, zumindest wenn die erhöhte Konzentration durch Atemluft bedingt ist.

Im weiteren Verlauf dieser Schülerumweltstudie ist geplant zu überprüfen, ob Bienen auch auf eine erhöhte TVOC-Konzentration reagieren, wobei der Aufbau es erlaubt, dass im nächsten Schuljahr weitere Schüler*innen eine Umweltstudie mit neuen Fragestellungen und neuen Sensoren durchführen.

\section{Literatur}

[1] Höfner, S., Schütze, A., Brück, B., Hirth, M., Kuhn, J. (2019). Citizen Science für Schüler*innen: Durchführung von Umweltstudien mit Smartphone und mobiler Messtechnik, Tagungsband, 20. GMA/ITG Fachtagung Sensoren und Messsyste, S. 476 - 481, doi: 10.5162/sensoren2019/6.1.5

[2] Wong, L.H., Looi, C.K. (2011). What seams do we remove in mobile assisted seamless learning? A critical review of the literature. Computers and Education, 57 (4), 2364-2381

[3] Storksdieck, M., Shirk, J.L., Cappadonna, J.L., Domroese, M., Göbel, C., Haklay, C., Miller-Rushing, A.J., Roetman, P., Sbrocchi, C., Vohland, K. (2016). Associations for citizen science: Regional knowledge, global collaboration. Citizen science: Theory and practice, 1(2), 10 http://dx.doi.org/10.5334/cstp.55

[4] Aceves-Bueno, E., Adeleye, A., Feraud, M., Huang, Y., Tao, M., Yang, Y., Anderson, S. (2017). The Accuracy of Citizen Science Data: A Quantitative Review. Bulletin of the Ecological Society of America. 98. 278-290. 10.1002/bes2.1336.

[5] Bonney, R., Cooper, C. B., Dickinson, J., Kelling, S., Phillips, T., Rosenberg, K.V., Shirk, J. (2009). Citizen science: A developing tool for expanding science knowledge and scientific literacy. BioScience, 59(11), 977-84. http://www.jstor.org/stable/27736031

[6] McDonough MacKenzie, C., Murray, G. Primack, R., Weihrauch, D. (2016). Lessons from citizen science: Assessing volunteer-collected plant phenology data with Mountain Watch. Biological Conservation. doi: 10.1016/j.biocon.2016.07.027

[7] Price, C. A., Lee, H.-S. (2013). Changes in participants' scientific attitudes and epistemological beliefs during an astronomical citizen science project. Journal of Research in Science Teaching, 50(7), 773-801.

[8] Ballard, H., Dixon, C., Harris, E. (2016). Youthfocused citizen science: Examining the role of environmental science learning and agency for conservation. Biological Conservation. doi: 10.1016/j.biocon.2016.05.024.
[9] Dahlstrom, E., Bichsel, J. (2014). ECAR Study of Undergraduate Students and Information Technology. Research report. Louisville, CO: ECAR

[10] Fies, C., Marshall, J. (2006) Classroom Response Systems: A Review of the Literature. J Sci Educ Technol 15: 101. doi: 10.1007/s10956-006-0360-1

[11] Fleuren, D. (2016). Open MINT Labs: Mit virtuellen Laboren zu höherem Lernerfolg. Junges Forum Medien und Hochschulentwicklung (JFMH13). Münster; New York: Waxmann 2016, S. 141-150 URN: urn:nbn:de:0111-pedocs-168213

[12] Kuhn, J., Vogt, P. (2015). Smartphone \& Co. in Physics Education: Effects of Learning with New Media Experimental Tools in Acoustics. In W. Schnotz, A. Kauertz, H. Ludwig, A. Müller and J. Pretsch (Eds.), Multidisciplinary Research on Teaching and Learning (pp. 253-269). Basingstoke, UK: Palgrave Macmillan

[13] Kuhn, J., Müller, A. (2014). Context-based science education by newspaper story problems: A study on motivation and learning effects, Perspectives in Science Volume 2, issues 1-4, Pages 5-21

[14] Mayer, R. E. (2002). Multimedia learning. Psychology of learning and motivation, 41, 85-139. doi: 10.1016/S0079-7421(02)80005-6

[15] Seeley T.D. (1977). Measurement of nest cavity volume by the honey bee (Apis mellifera L.). Behav. Ecol. Sociobiol. 2: 201-227.

[16] Starks, O.T., Blackie, C.A., Seeley T.D. (2000). Fever in honeybee colonies. Naturwiss. 87: 229-23

[17] Sachs, R., Tautz, J., (2017). How Bees (Apis Mellifera) Reduce Humidty in the Beehive by Means of Active Ventilation. https://www.researchgate.net/publication/315083892 _How_Bees_Apis_Mellifera_Reduce_Humidity_in_th è_Beehive_by_Means_of_Active_Ventilation

[18] Negri, I., Mavris, C., Di Prisco, G., Caprio, E., Pellecchia, M., (2015). Honey Bees (Apis meelfera, L.) as Active Samplers of Airborne Particulate Matter. PLoS ONE 10(7): e0132491. https://doi.org/10.1371/journal.pone.0132491

[19] Keith M. Doull (1976). The effects of different humidites on the hatching of eggs of honeybees. Apidologie, Springer Verlag, 1976, 7 (1), pp.61-66, doi:10.1051/apido:19760104

[20] Umweltbundesamt, Grenzwerte für Feinstaub, https://www.umweltbundesamt.de/daten/luft/feinstau b-belastung (abgerufen am 12.11.2019)

\section{Danksagung}

Diese Arbeiten wurden durch die Deutsche Bundesstiftung Umwelt (DBU) im Rahmen der Förderlinie Bildung für nachhaltige Entwicklung (BNE) gefördert.

Ein besonderer Dank gilt Dr. Martin Wörner, der als Mitglied des Landesverbands saarländischer Imker diese Umweltstudie durch das Zurverfügungstellen der Bienenvölker und -körbe ermöglicht hat und durch seine Expertise und Anregungen maßgeblich zum Erfolg beigetragen hat. 